\title{
Does the appearance of the cutaneous scar after cesarean section reflect the residual myometrial thickness?
}

\author{
Ammar Al Naimi ${ }^{1,2}$ D $\cdot$ Niki Mouzakiti $^{1} \cdot$ Carmen Eißmann $^{1} \cdot$ Frank Louwen $^{2} \cdot$ Franz Bahlmann $^{1}$
}

Received: 26 October 2020 / Accepted: 15 December 2020 / Published online: 7 January 2021

(c) The Author(s) 2020

\begin{abstract}
Purpose The aim of this study is to utilize the Manchester scar scale (MSS) and ultrasound in investigating the association between uterine wall defects and cutaneous scar characteristics after cesarean section (CS).

Methods This is a prospective cohort study. The degree of myometrial loss was quantified by calculating a residual myometrial thickness (RMT) ratio as a percentage of RMT to the pre-cesarean anterior uterine wall thickness. Cutaneous scar assessment was performed according to the MSS. Spearman's correlation and the Kruskal-Wallis test with a cut-off value of $p<0.05$ were used for statistical analysis.

Results Two hundred forty seven women, of which 2.4\% had an Asian, 3.6\% an Afro-American, 82\% a Caucasian and $12 \%$ a Mediterranean background, were recruited. The RMT ratio ranged between 11.9 and $100 \%$ with a median of $55.8 \%$ and an average of 56\%. MSS scores ranged from 4 to 13 with a median of 5 and an average of 6 . Spearman's correlation between MSS and RMT ratio show a rho of -0.01 with a $p$ value of 0.8 . The correlation between MSS and RMT ratio within the four ethnical groups showed a $p$ value between 0.3 and 0.8 and a rho between 0.8 and -0.8 . The Kruskal-Wallis test showed an eta ${ }^{2}$ of 0.13 and a $p$ value of 0.0002 for the effect of ethnicity on MSS and an eta ${ }^{2}$ of 0.009 and a $p$ value of 0.68 for the effect of ethnicity on the RMT ratio.

Conclusion CS laparotomy scars heal differently between ethnical groups, but generally with satisfying results. Ethnicity does not affect myometrial healing and scar appearance does not reflect myometrial healing after CS. Thus, separate uterine sonographic assessment is recommended.
\end{abstract}

Keywords Manchester scar scale $\cdot$ Cesarean section $\cdot$ Ultrasound $\cdot$ Residual myometrial thickness

\section{Introduction}

Due to the worldwide increase of cesarean section (CS) rate to about $30 \%$ in developed countries, CS with a suprapubic (Pfannenstiel-Kerr) incision has become the most common abdominal surgery for women [1]. These laparotomy scars heal relatively well, and their anatomical position makes them easy to hide if healing is not cosmetically satisfactory.

Ammar Al Naimi

ammar.alnaimi@uclmail.net

1 Department of Obstetrics and Gynecology, Dr. Senckenberg Foundation, Buergerhospital, Nibelungenallee 37-41, 60318 Frankfurt am Main, Hessen, Germany

2 Department of Obstetrics and Gynecology, University Hospital, Goethe University Frankfurt am Main, Theodor-Stern-Kai 7, Frankfurt am Main 60590, Hessen, Germany
Therefore, for a surprisingly long time obstetricians tended to ignore the importance of this scarring [2]. Deformed scarring has psychological effects and can lead to social dysfunction, communication hurdles and self-confidence issues. In addition to this distress, scarring can cause physical discomfort, pain, and pruritus [3]. With increasing focus on wound healing, several tools have been devised to objectively describe scar healing either quantitatively or qualitatively, and these tools have been utilized for assessing cutaneous scarring after a CS [4]. One of these assessment tools that are popular for objectively assessing different cutaneous scars is the Manchester Scar Scale (MSS) [5].

Uterine wall defects at the CS site are common [6], and several studies showed the association between these defects and gynecological symptoms such as spotting and chronic pelvic pain $[7,8]$. Vaginal ultrasound represents the gold standard for assessing uterine wall defects after CS [9]. The aim of this study is to utilize ultrasound and MSS in investigating the 
association between the severity of uterine wall defects and the characteristics of the cutaneous scars after CS.

\section{Materials and methods}

This is a prospective cohort study where women with a history of only one CS were recruited 12-24 months postoperatively. Inclusion criteria are age above 18 , gestational age at delivery between $24+0$ and $42+0$ weeks, elective, unplanned and emergency CS, and signing a consent form. Exclusion criteria were a history of two CSs or more, a history of vertical uterotomy, and a history of additional uterine surgery. The myometrial scar assessment was performed with vaginal ultrasound as shown in Fig. 1. Sonographic volumetric datasets from each patient, where the uterus was completely visualized, were acquired with a $5-13 \mathrm{MHz}$ micro-convex transvaginal transducer, GE RIC6-12-D (Voluson E10, GE Healthcare GmbH, Munich, Germany). The desired planes for evaluating the myometrial defects at the CS scar were acquired with multiplanar views. The residual myometrial thickness (RMT) was measured according to the recommendations of Jordans et al. [9]. The depth of the myometrial loss (D) at the CS scar was measured on the sagittal plane perpendicular to the endometrial line. The degree of myometrial loss was quantified by calculating an RMT ratio as a percentage of RMT to the assumed original pre-cesarean anterior uterine wall thickness. The formula utilized for this calculation is RMT ratio $=\mathrm{RMTx} 100 /$ $(\mathrm{RMT}+\mathrm{D})$.

Cutaneous scar assessment was performed according to the MSS. Assessment was carried out by a member of the study team who was not involved with the operation to avoid bias. The MSS evaluates and scores four parameters including scar color (perfect match, slight-, obvious-, or gross- mismatch with surrounding skin), surface shine or appearance (matte or shiny), contour (flush with surrounding skin, slightly indented, hypertrophic or keloid), and distortion (none, mild, moderate or severe). The score for each parameter begins with 1 and increases up to 4 according to the quality of the characteristic. The range of the final score is between 4 and 14 where lower values denote a better healing outcome, while a high total score indicates abnormal scarring [5]. A tabular presentation of MSS is shown in Table 1.

Spearman's correlation test was used to assess the correlation between the individual MSS and RMT ratio. Patients were further subdivided into ethnical groups in order to

Table 1 Manchester Scar Scale (MSS) with the assessed parameters and their corresponding value [5]

\begin{tabular}{lll}
\hline MSS parameters & Category & Points \\
\hline Scar color & Perfect match & 1 \\
& Slight mismatch & 2 \\
& Obvious mismatch & 3 \\
Surface shine & Gross mismatch & 4 \\
& Matte & 1 \\
Contour & Shiny & 2 \\
& Flush with surrounding skin & 1 \\
& Slightly indented & 2 \\
Distortion & Hypertrophic & 3 \\
& Keloid & 4 \\
& None & 1 \\
Outcome & Mild & 2 \\
& Moderate & 3 \\
& Severe & 4 \\
& Best & $(4-$ \\
& Worst & $14)$ \\
\hline
\end{tabular}

Fig. 1 Transvaginal ultrasound with uterine sagittal view demonstrating how RMT ratio was measured. arrow: the myometrial defect in the form of a niche; $D$ the depth of the myometrial loss, $R M T$ residual myometrial thickness. RMT ratio $=\mathrm{RMT} \times 100 /(\mathrm{RMT}+\mathrm{D})$

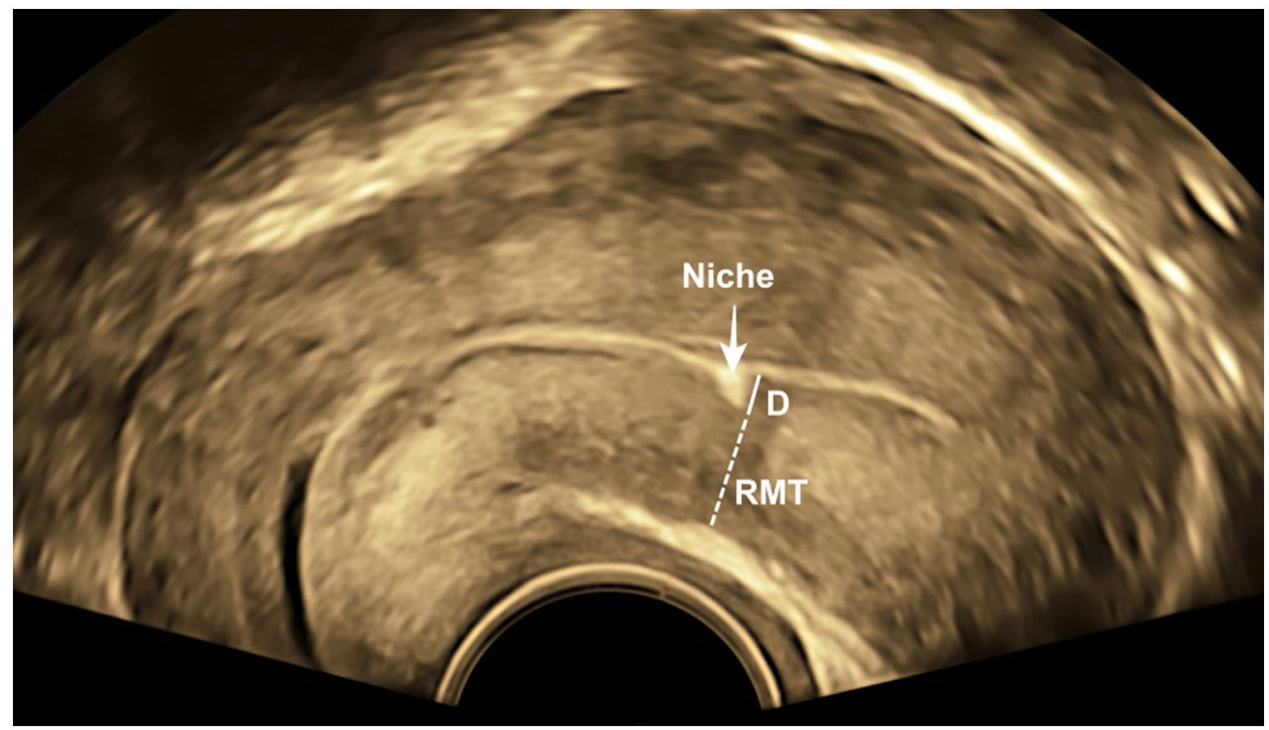


investigate the effect of ethnicity on both MSS score and RMT ratios with the Kruskal-Wallis test. The cut-off value of $p$ below 0.05 was considered to be significant.

\section{Results}

This study included 247 women, with a 2.4\% Asian, 3.6\% Afro-American, $82 \%$ Caucasian and 12\% Mediterranean background. The average point in time of recruitment was 18 months postoperatively. The RMT ratio ranged between 11.9 and $100 \%$ with a median of $55.8 \%$ and an average of $56 \%$. The range of MSS scores for this cohort was between 4 and 13 with a median of 5 and an average of 6 (approximated from 5.82). Figure 2 shows the two extremes of the MSS for this study cohort.

Spearman's correlation test between MSS and RMT ratio showed a rho of -0.01 with a $p$ value of 0.8 . The $p$ value remained high between 0.3 and 0.8 and the rho ranged between 0.8 and -0.8 when the correlation between RMT ratio and MSS was tested within the separate ethnical groups. The Kruskal-Wallis test for the effect of ethnicity

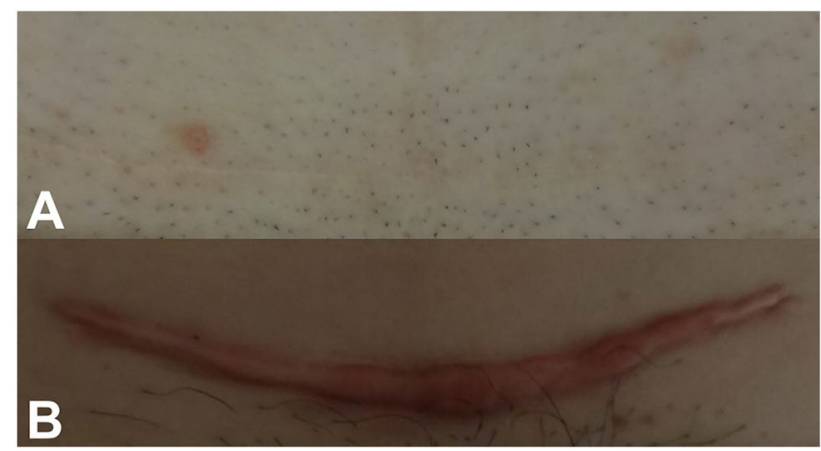

Fig. 2 Photographic appearance of Cesarean section laparotomy scar showing a perfect healing with MSS 4 and b severe scarring with MSS 13

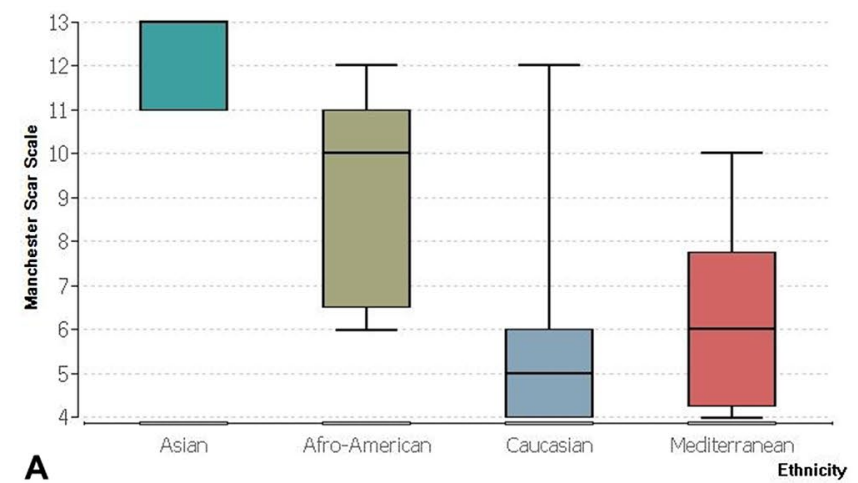

on MSS showed an eta ${ }^{2}$ of 0.13 and a $p$ value of 0.0002 . The effect of ethnicity on the RMT ratio showed an eta ${ }^{2}$ of 0.009 and a $p$ value of 0.68 . The differences between both the MSS scores and the RMT ratio among the ethnical groups is demonstrated in Fig. 3.

\section{Discussion}

Wound healing in adult humans can invariably cause scarring and subsequently have an adverse effect on both function and cosmetic appearance. The process of wound healing is governed by genetic, immunological, and hormonal factors that lead to tissue regeneration. The phases of exudation, resorption, and regeneration during wound healing are mutual between the cutaneous and serosal wounds and the myometrium is also expected to be associated with skin healing [10]. The characteristics of a cutaneous CS scar have shown an association with intraabdominal adhesions during repeat CS [11]. While the assessment of adhesions is difficult to quantify, objective quantifiable myometrial CS scar assessment is possible in a non-gestational state. Thus, we decided to implement two quantitative methods for assessing intraindividual healing results of both the cutaneous scar and the myometrial scar post-cesarean. A Delphi-based guideline was published in 2019 to standardize the sonographic measurement methodology of CS scars [9]. The measurements of our study were based on this guideline, and in order to account for individual uterine size differences between different women the RMT ratio was utilized [12]. Other studies employed similar methods and decided to assess CS scars with ratios instead of utilizing absolute values. The myometrial thinning at the scar was measured as a deficiency ratio and a ratio above $50 \%$ was considered to be severe. Our proposed RMT ratio has an inverse relationship to such a deficiency ratio and a higher RMT ratio reflects better healing [13]. Our data show both median and average RMT

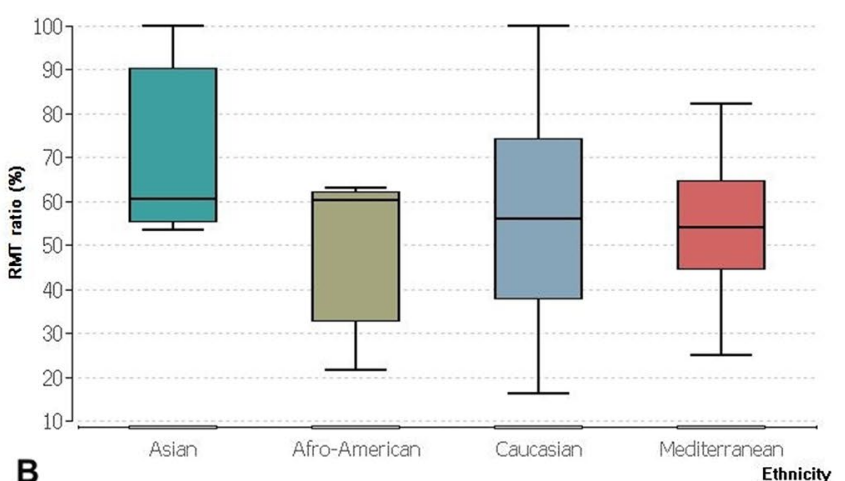

Fig. 3 Box plot showing the medians and distribution of a MSS and b RMT ratio depending on ethnicity 
ratios of around $56 \%$ which contradicts the assumption that a deficiency above $50 \%$ is severe.

The diverse variety of surgical techniques involved with CS and the importance of evidence-based practices regarding skin closure have led to increasing research interest for evaluating CS procedures [14]. Surgical wound complications and infections are the most common adverse outcomes after CS with an incidence between $4.9 \%$ and $9.8 \%$ and collectively represent the most costly complication of CS [15]. In an attempt to define important criteria for assessing wound healing, clinicians rely on both cosmetic and functional parameters [16]. While the MSS is based mainly on cosmetic criteria, its score correlates directly with the histological features of the scar tissue. It is objective, reproducible, valid, uncostly in both time and manpower, and simple to implement [5] Therefore, it is one of the most popular scores among clinicians [17]. Therefore, we opted to implement the MSS for this study also, and our median and averages scores of 5 and 6 , respectively, show that this cohort generally exhibited good wound healing. We could have attributed this good healing to the usage of sutures instead of clips for skin closure, but randomized controlled trials have shown otherwise. They conclude that the cosmetic appearance of the cutaneous CS scars is comparable regardless of the skin closure technique. Therefore, a patient's own healing potential was attributed as the significant influencing factor [2]. It is safe to assume that all wounds in our cohort, both cutaneous and myometrial, had reached their full healing potential due to the average recruitment time of 18 months postoperatively. It is known that cutaneous wounds finish healing within six months and myometrial CS scars reach their healing potential between 6 and 9 months post-cesarean and remain constant up to a following pregnancy [18].

Both myometrial and cutaneous CS scars are the product of wound-healing processes, thus they share similar biologic healing pathways [19]. Transforming growth factor $\beta$ (TGF- $\beta$ ) plays a pivotal role in the process of wound healing. TGF- $\beta$ induces the production of collagen fibers and TGF- $\beta$ overexpression leads to abundance of collagen that results in hypertrophic scars and keloid formation [20]. Altered or reduced expression of TGF- $\beta$ on the other hand leads to impaired collagen deposition in the scar tissue and results in abnormal scar formation or scar dehiscence of the lower uterine segment [21]. Therefore, we expected to show a negative correlation between MSS and RMT ratio for our patients. Our data were unable to support this theory as the correlation between RMT and MSS showed a statistically insignificant $p$ value of 0.8 . Even with further subdivision of the cohort into ethnical groups, a correlation between MSS and RMT could not be demonstrated statistically, which leads us to reject our proposed correlation between the two. Several studies attributed the increased collagen production during healing as the main reason for the association between postoperative high cutaneous MSS values and intraabdominal adhesions [22]. This mechanism does not apply to the myometrial healing in this cohort.

Ethnicity affects cutaneous scar healing significantly. Individuals from Asian, Afro-American, and Mediterranean backgrounds possess the so called 'ethnic skin', which is highly susceptible to scarring and keloid formation [23]. This fact is evident in our cohort as Asian, Afro-American, and Mediterranean patients had significantly higher MSS scores compared to Caucasian women and the Kruskal-Wallis results prove this effect with an eta ${ }^{2}$ of 0.13 and a $p$ value of 0.0002 . The RMT ratio, however, was unaffected by ethnicity and Fig. 3 shows very close RMT ratio medians for the four selected groups. Myometrial healing is a complex process which is not only governed by genotypic, but also phenotypic factors and leads to heterogenous behaviors of myometrial surgical wounds [21]. While several factors can affect scar healing, individual healing potential varies from one person to another and plays a significant role in cutaneous scarring [24]. One limitation to our study is the uneven distribution of the different ethnicities among the cohort which might cause bias.

In conclusion, even though there are differences in the appearance of the cutaneous CS scar between ethnical groups, these scars usually heal with satisfying results. These scars do not represent the myometrial healing after CS and separate uterine sonographic assessment is recommended for future counseling. Ethnicity does not affect the myometrial healing and further research is needed to identify influencing factors.

Acknowledgements This work was supported by the Dr. Senckenberg Foundation.

Author contributions A.AN: project development, data collection, manuscript writing. N.M: data analysis. C.E: data collection. F.L: manuscript editing. F.B: project development.

Funding Open Access funding enabled and organized by Projekt DEAL.

\section{Compliance with ethical standards}

Conflict of interest All authors declare no conflicts of interest.

Ethics approval The study has been approved by the Ethics Committee at the Hesse State Chamber of Physicians.

Consent to participate Written consent is within the inclusion criteria.

Consent for publication Included in the participation consent.

Open Access This article is licensed under a Creative Commons Attribution 4.0 International License, which permits use, sharing, adaptation, distribution and reproduction in any medium or format, as long as you give appropriate credit to the original author(s) and the source, provide a link to the Creative Commons licence, and indicate if changes were made. The images or other third party material in this article are included in the article's Creative Commons licence, unless indicated 
otherwise in a credit line to the material. If material is not included in the article's Creative Commons licence and your intended use is not permitted by statutory regulation or exceeds the permitted use, you will need to obtain permission directly from the copyright holder. To view a copy of this licence, visit http://creativecommons.org/licenses/by/4.0/.

\section{References}

1. Betran AP, Ye J, Moller A-B, Zhang J, Gulmezoglu AM, Torloni MR (2016) The Increasing trend in caesarean section rates: global, regional and national estimates: 1990-2014. (Report). PLoS ONE 11:2. https://doi.org/10.1371/journal.pone.0148343

2. Cromi A, Ghezzi F, Gottardi A, Cherubino M, Uccella S, Valdatta L (2010) Cosmetic outcomes of various skin closure methods following cesarean delivery: a randomized trial. Am J Obstet Gynecol 203(1):36.e31-36.e38. https://doi.org/10.1016/j. ajog.2010.02.001

3. Brown BC, McKenna SP, Siddhi K, McGrouther DA, Bayat A (2008) The hidden cost of skin scars: quality of life after skin scarring. J PlastReconstrAesthetSurg 61(9):1049-1058. https:// doi.org/10.1016/j.bjps.2008.03.020

4. Mackeen AD, Fleisher J, Khalifeh A, Pettker CM, Berghella V (2014) Patient satisfaction and cosmetic outcome in a randomized study of cesarean skin closure. ObstetrGynecol (NY 1953) 123(Suppl 1):4S-5S. https://doi.org/10.1097/01.AOG.0000447248 .54066 .04

5. Beausang E, Floyd H, Dunn KW, Orton CI, Ferguson MWJ (1998) A new quantitative scale for clinical scar assessment. PlastReconstrSurg 102(6):1954-1961. https://doi.org/10.1097/00006534199811000-00022

6. VikharevaOsser O, Jokubkiene L, Valentin L (2009) High prevalence of defects in Cesarean section scars at transvaginal ultrasound examination. Ultrasound ObstGyn 34(1):90-97. https://doi. org/10.1002/uog.6395

7. Wang H-Y, Jiang Q, Shi H, Xu Y-Q, Shi A-C, Sun Y-L, Li J, Ning Q, Shen G-X (2016) Effect of caesarean section on maternal and foetal outcomes in acute fatty liver of pregnancy: a systematic review and meta-analysis. Scie Rep (Nat Publ Group) 6(1):28826. https://doi.org/10.1038/srep28826

8. Thurmond AS, Harvey WJ, Smith SA (1999) Cesarean section scar as a cause of abnormal vaginal bleeding: diagnosis by sonohysterography. J Ultras Med 18(1):13-16. https://doi.org/10.7863/ jum.1999.18.1.13

9. Jordans I, De Leeuw R, Stegwee S, Amso N, Barri-Soldevila P, Van Den Bosch T, Bourne T, Brölmann H, Donnez O, Dueholm M (2019) Sonographic examination of uterine niche in non-pregnant women: a modified Delphi procedure. Ultrasound Obstet Gynecol 53(1):107-115

10. Brochhausen C, Schmitt VH, Planck CN, Rajab TK, Hollemann D, Tapprich C, Krämer B, Wallwiener C, Hierlemann H, Zehbe R, Planck H, Kirkpatrick CJ (2012) Current strategies and future perspectives for intraperitoneal adhesion prevention. J GastrointestSurg 16(6):1256-1274. https://doi.org/10.1007/s 1160 5-011-1819-9

11. Dogan NU, Haktankacmaz SA, Dogan S, Ozkan O, Celik H, Eryilmaz OG, Doganay M, Gulerman C (2011) A reliable way to predict intraabdominal adhesions at repeat cesarean delivery: scar characteristics. Acta Obstet GynecolScand 90(5):531-534. https ://doi.org/10.1111/j.1600-0412.2011.01080.x

12. Al Naimi A, Mouzakiti N, Hondrich M, Louwen F, Bahlmann F (2020) The B-mode sonographic evaluation of the post-caesarean uterine wall and its methodology: a study protocol. J Obstet Gynaecol Res. https://doi.org/10.1111/jog.14492

13. Seliger G, Muendane A, Chaoui K, Hiller GGR, Lautenschläger C, Costa S-D, Tchirikov M (2018) Does ultrasound-guided intervention during repeat cesarean sections improve uterine scar architecture and reduce the number of scars? A prospective controlled clinical intervention trial. J Perinat Med 46(8):857. https://doi. org/10.1515/jpm-2017-0394

14. Tully L, Gates S, Brocklehurst P, McKenzie-McHarg K, Ayers S (2002) Surgical techniques used during caesarean section operations: results of a national survey of practice in the UK. Eur $\mathbf{J}$ Obstet Gyn R B 102(2):120-126. https://doi.org/10.1016/S0301 $-2115(01) 00589-9$

15. Sandy-Hodgetts K, Parsons R, Norman R, Fear MW, Wood FM, White SW (2020) Effectiveness of negative pressure wound therapy in the prevention of surgical wound complications in the cesarean section at-risk population: a parallel group randomised multicentre trial-the CYGNUS protocol. BMJ Open 10(10):e035727. https://doi.org/10.1136/bmjopen-2019-035727

16. van de Kar AL, Corion LU, Smeulders MJ, Draaijers LJ, van der Horst CM, van Zuijlen PP (2005) Reliable and feasible evaluation of linear scars by the patient and observer scar assessment Scale. PlastReconstrSurg 116(2):514-522. https://doi.org/10.1097/01. prs.0000172982.43599.d6

17. Owji N, Khademi B, Khalili MR (2018) Effectiveness of topical onion extract gel in the cosmetic appearance of blepharoplasty scar. J ClinAesthetDermatol 11(10):31-35

18. Baranov A, Salvesen K $\AA$, Vikhareva O (2017) Assessment of cesarean hysterotomy scar before pregnancy and at 11-14 weeks of gestation: a prospective cohort study. Ultrasound Obstet Gynecol 50(1):105-109

19. Bielefeld KA, Amini-Nik S, Alman BA (2013) Cutaneous wound healing: recruiting developmental pathways for regeneration. Cell Mol Life Sci 70(12):2059-2081. https://doi.org/10.1007/s0001 8-012-1152-9

20. Gallant-Behm CL, Olson ME, Hart DA (2005) Cytokine and growth factor mRNA expression patterns associated with the hypercontracted, hyperpigmented healing phenotype of red duroc pigs: a model of abnormal human scar development? J Cutan Med Surg 9(4):165-177. https://doi.org/10.1007/s10227-005-0105-4

21. Lofrumento DD, Di Nardo MA, De Falco M, Di Lieto A (2017) Uterine wound healing: a complex process mediated by proteins and peptides. Curr Protein Pept Sci 18(2):125-128. https://doi. org/10.2174/1389203717666160322145939

22. Kokanalı D, Kokanalı MK, Topcu HO, Ersak B, Tascı Y (2019) Are the cesarean section skin scar characteristics associated with intraabdominal adhesions located at surgical and non-surgical sites. J Gynecol Obstet Hum 48(10):839-843. https://doi. org/10.1016/j.jogoh.2019.03.026

23. Madu P, Kundu RV (2014) Follicular and scarring disorders in skin of color: presentation and management. Am J ClinDermatol 15(4):307-321. https://doi.org/10.1007/s40257-014-0072-x

24. Bayat A, McGrouther DA, Ferguson MW (2003) Skin scarring. BMJ 326(7380):88-92. https://doi.org/10.1136/bmj.326.7380.88

Publisher's Note Springer Nature remains neutral with regard to jurisdictional claims in published maps and institutional affiliations. 